\title{
Réimplantation de cellules génétiquement modifiées dans des néo-organes vascularisés
}

La plupart des protocoles de thérapie génique prévoient que les cellules à modifier soient manipulées ex vivo puis réimplantées dans l'organisme où elles cxprimeront leur nouvelle fonction. Cependant, il n'est guère que les cellules hématopoïétiques qui, lorsqu'elles sont réintroduites par simple réinjection, trouvent leur chemin vers un stroma où clles pourront croître et se multiplier. Les autres cellules, qui ne possèdent pas à leur surface de récepteur de domiciliation (homing receptor), doivent être réimplantées en des sites particuliers. Une matrice extraccllulaire propice à leur attachement est en effet néccssaire à leur survie, qui dépend également de facteurs trophiques produits par les cellules voisines ou apportés par le système vasculaire. Injectés dans la circulation ou dans la cavité péritonéale, hépatocytes, myoblastes, cellules endothéliales ou fibroblastes essaiment avec une très faible efficacité et disparaissent pour la plupart.

Différentes méthodes d'implantation en des sites spécifiques de cellules génétiquement modifiées ont été utilisées avec succès. Les hépatocytes peuvent êtrc injectés dans la veine porte ou dans la rate [1-3] et les myoblastes dans le muscle squelettique [4]. Les cellules endothéliales vasculaires sont utilisées pour recouvrir un greffon artériel artificiel [5] et des structures transplantables équivalentes au derme peuvent être constituées in vitro avec des fibroblastes [6, 7]. Les fibroblastes peuvent également être greffés à l'intérieur du cortex cérébral où ils survivent pendant plusieurs semaines [8].

Ces méthodes de réimplantation in situ conduisent à l'établissement irréversible d'un implant diffus de ccllules génétiquement modifiées dans l'organe ou le tissu. Il est en général difficile de surveiller directement l'ćvolution de cet implant et d'en évaluer la survie. D'autre part, les protocoles compren- nent souvent des étapes traumatiques (résection d'un lobe du foie, lésion du muscle), qui sont destinées à provoquer une regénération de l'organe. L'environnement physiologique obtenu dans ces conditions est en effet beaucoup plus favorable à l'implantation des cellules modifiées génétiquement. Ces manœuvres pourraient être évitées s'il était possible de réimplanter les cellules dans une structure autonome et vascularisée comportant tous les éléments matriciels et nutritifs nécessaires. Ce type d'implant localisable et accessible serait assimilable à une chambre de diffusion. Des travaux récents indiquent qu'il est possible d'obtenir de telles structures et d'y installer des fibroblastes génétiquement modifiés qui s'y maintiennent et expriment le produit du transgène pendant plusieurs mois.

De nombreuses équipes ont cherché à pallier les difficultés de la transplantation cellulaire en développant des supports synthétiques qui permettent l'attachement et la survie des cellules. Des micro-billes de dextran peuvent être utilisées pour la culture puis l'implantation intrapéritonéale d'hépatocytes [9] et permettent d'augmenter la période de survie de ces cellules. On a également cherché, avec des succès variables, à introduire différents types cellulaires dans des réceptacles synthétiques [10]. Ce type d'implant provoque généralement une importante réaction inflammatoire.

En 1988, l'équipe de Thomas Maciag au NIH (Bethesda, MD, USA) a montré qu'il était possible d'induire une angiogenèse limitée en introduisant à l'intérieur de la cavité péritonéale une éponge de collagène de type I imprégnée de facteur de croissance fibroblastique (aFGF) [11]. Des hépatocytes établis en lignée continue avaient pu être inclus dans cette structure, conduisant à la formation d'un implant récupérable après six semaines. La même équipe a par la suite utilisé des fibres synthétiques de polytétrafluoroéthylène (PTFE), un composé inerte largement utilisé en chirurgie, comme support pour le collagène et le FGF. Ces fibres, implantées dans la cavité péritonéale chez le rat, permettent le développement en quelques semaines de structures individualisées, baptisées organoïdes ou néo-organes ([12] et $\mathrm{m} / \mathrm{s}$ $n^{\circ} 2$, vol. 6, p. 144). On y trouve une abondante vascularisation qui irrigue un tissu principalement constitué de fibroblastes. Des cellules musculaires lisses, et même des éléments de tissu nerveux peuvent y être observés. Thompson et al. ont utilisé ces organoïdes chez le rat Gunn comme réceptacles pour des hépatocytes primaires. Cet animal présente un déficit du système hépatique d'élimination de la bilirubine, produit de dégradation de l'hème dont l'accumulation excessive est toxique pour le système nerveux central. La publication décrit l'injection d'hépatocytes prélevés sur des rats congéniques normaux Wistar, à l'intérieur d'organoïdes formés chez le rat Gunn. Une réduction marquée du taux de bilirubine sérique aurait été observée. Ces résultats spectaculaires n'ont jamais été reproduits et les auteurs en ont récemment demandé le retrait [13]. Les observations concernant la constitution des néo-organes n'en restent pas moins valables, bien que l'évolution à long terme de ces structures n'ait pas été maîtrisée au cours de ces premières études. Les conditions utilisées semblent conduire à une réaction inflammatoire importante qui évolue en fibrose après quelques semaines.

Dans le but d'obtenir des structures permettant la réimplantation et la survie de cellules génétiquement modifiées, notre équipe a cherché à améliorer le maintien des néo-organes en assurant un meilleur revêtement des 
fibres synthétiques par le collagène et en utilisant diverses sources de matrice extracellulaire et de facteurs de croissance. Dans un premier temps, nous sommes parvenus à constituer des néoorganes richement vascularisés dans la cavité péritonéale de souris. Ces structures sont stables pendant plus de six mois, très peu inflammatoires et n'évoluent pas vers la fibrose. Environ trois millions de fibroblastes dermiques autologues peuvent être injectés dans l'organoïde. Ils s'établissent dans le tissu conjonctif lâche et y résident pendant plusieurs mois. Nous avons utilisé cette méthode pour distribuer de manière continue le produit d'un transgène in vivo. A partir d'une biopsie cutanée, des fibroblastes ont été établis en culture primaire et ont reçu l'ADNc humain codant pour la $\beta$ glucuronidase porté par un vecteur rétroviral. Ils ont ensuite été introduits dans un organoïde préformé dans la cavité péritonéale d'une souris. La $\beta$ glucuronidase est une enzyme lysosomiale qui est en partie sécrétée et dont la forme extracellulaire peut être captée à distance par des cellules possédant les récepteurs membranaires adéquats. Ceux-ci sont spécifiques des macrophages (récepteur au mannose/Nacétyl glucosamine) ou portés par la plupart des cellules (récepteur au mannose-6-phosphate). L'enzyme purifiée administrée par voie intraveineuse est captée par différents organes [14]. Nos expériences indiquent que l'enzyme sécrétée dans le sérum par un implant cellulaire se comporte de manière semblable. Trois mois après l'implantation des fibroblastes génétiquement modifiés dans des organoïdes, l'enzyme humaine a été retrouvée au niveau du foie, de la rate et des poumons des animaux porteurs [15].

Nous avons, dans un second temps, simplifié cette technique de réimplantation de fibroblastes possédant le transgène de façon à augmenter la quantité de cellules transplantées, tout en procédant en un seul temps opératoire. Désormais, les fibroblastes ne sont plus injectés dans l'organoïde préformé, mais directement inclus dans un gel de collagène contenant les fibres synthétiques et les facteurs de croissance. Ce gel d'un volume initial de deux à trois millilitres contient 15 à 20 millions de cellules qui s'attachent au collagène, provoquant la formation d'un maillage dense. L'ensemble se rétracte rapidement in vitro, peut être réimplanté dans la cavité péritonéale au bout de quelques jours et conduit à la formation d'un organoïde stable. Nous avons utilisé ce procédé pour introduire des fibroblastes sécrétant de la $\beta$-glucuronidase chez des souris déficitaires pour cette enzyme. Ces animaux présentent une mucopolysaccharidose de type VII, identique à la maladie de Sly chez l'homme, avec de nombreuses atteintes squelettiques et neurologiques et de graves signes de surcharge lysosomiale dans les principaux organes [16]. La distribution de l'enzyme à partir de l'implant intrapéritonéal a été observée dans une série de quinze souris atteintes de la maladie, pendant une période de six mois. Elle conduit à une disparition de la surcharge dans le foie et la rate des animaux traités.

Ces résultats indiquent qu'une protéine thérapeutique peut-être distribuée in vivo à partir d'un néo-organe, pendant une durée prolongée. De nombreuses situations pathologiques humaines pourraient bénéficier d'une approche de ce type. L'implantation chez un patient d'un ou plusieurs organoïdes contenant quelques centaines de millions de fibroblastes génétiquement modifiés pour produire une enzyme lysosomiale, une hormone, un facteur de coagulation ou une protéine antivirale ne devrait pas comporter de difficulté majeure. L'opération n'impliquerait que des techniques chirurgicales relativement peu invasives; l'implant cellulaire confiné au néoorgane serait facile à surveiller et, si besoin est, à retirer. Dans cette perspective d'application clinique, nos travaux chez la souris et le chien cherchent maintenant à documenter de façon plus détaillée la longévité de l'implant et le comportement à très long terme des fibroblastes transplantés

Olivier Danos
Philippe Moullier
Jean-Michel Heard
Laboratoire rétrovinus et transfert génétique, Ins-
titut Pasteur, 28, rue du docteur Roux,
75724 Paris Cedex 15, France.

RÉFÉRENCES

1. Chowdhury JR, Grossman M, Gupta S, Chowdhruy NR, Baker JR, et al. Long-term improvement of hypcrcholesterolemia after exvivo gene therapy in LDLR-deficient rabbits. Science 1991; 254 : 1802-5.

2. Grossman M, Raper SE, Wilson JM. Transplantation of genetically modified autologous hepatocytes into non human primates : Feasibility and short-term toxicity. Hum Gene Ther $1992 ; 3$ : 501-10.

3. Kay MA, Baley P, Rothenberg S, Leland F, Fleming L, et al. Expression of human $\alpha$-antitrypsine in dogs after autologous transplantation of retroviral transduced hepatocytes. Proc Natl Acad Sci USA 1992; 89 : 89-93.

4. Dai Y, Roman M, Naviaux RK, Verma IM. Gene therapy via primary myoblasts: Long-term expression of factor IX protein following transplantation in vivo. Proc Natl Acad Sci USA 1992; 89 : 10892-5.

5. Wilson JM, Birinyi LK, Salomon RN, Libby $\mathrm{P}$, Callow $\mathrm{AD}$, et al. Implantation of vascular grafts lined with genetically modificd endothelial cells. Science $1989 ; 244$ : 1344-6.

6. Palmer TD, Rosman GJ, Osborne WRA, Miller AD. Genetically modified skin fibroblasts persist long after transplantation but gradually inactivate introduced genes. Proc Natl Acad Sci USA 1991; 88 : 1330-4.

7. Scharfmann R, Axclrod JH, Verma IM. Long-term in vivo expression of retrovirusmediated gene transfer in mouse fibroblast implants. Proc Natl Acad Sci USA 1991; 88 : 4626-30.

8. Gage FH, Kawaja MD, Fisher IJ. Genetically modified cells : applications for intracercbral grafting. Trends Neurosci 1991; 14 : 328-33.

9. Demetriou AA, Whiting JF, Feldman D, Levension SM, Chowdhury NR, et al. Replacement of liver function in rats by transplantation of microcarrier-attached hepatocytes. Science $1986 ; 233$ : 1190-2.

10. Wiktor-Jedrzcjzak WA, Bartocci A, Ferrante AW, Ahmed-Ansari A, Sell $\mathrm{K}$, et al. Total absence of colony-stimulating factor 1 in the macrophagc-deficient osteopetrosic (op/op) mouse. Proc Natl Acad Sci USA 1990 ; 87 : 4828-32.

11. Thompson JA, Anderson KD, Di Pictro JM, Zwicbel JA, Zametta M, et al. Sitedirected neovessel formation in vivo. Science $1988 ; 241$ : 1349-52.

12. Thompson JA, Haudenschild CC, Anderson $\mathrm{KD}$, Di Pietro JM, Anderson WF, et al. Heparin-binding growth factor 1 induces the formation of organoid ncovascular structures in vivo. Proc Natl Acad Sci USA 1989; 86 : 7928-32. 


\section{RÉFÉRENCES}

13. Thompson JA, Haudenschild CC, Anderson $\mathrm{KI}$, I Di Pictro JM, Anderson WF, et al. Heparin-binding growth factor 1 induces the formation of organoid ncovascular structures in vivo. Correction. Proc Natl Acad Sci USA $1992 ; 89$ : 7849.

14. Sly WS. Multiple recognition of human $\beta$-glucuronidase and their pinocytosis receptors : implications for enzyme therapy. Birth Defects $1980 ; 16: 115-28$.

15. Moullicr P, Maréchal V, Danos $O$, Heard JM. Continous systemic secretion of a lysosomal cnzyme by genctically-modificd mousc skin fibroblasts. Transplantation 1993 (sous presse).

16. Vogler C, Birkenmeicr EH, Sly WS, Levy B, Pegors C, et al. A murine model of mucopolysaccharidosis VII : gross and microscopic findings in beta-glucuronidasc-deficient micc. Am J Pathol 1990 ; 136 : 207-17

TIRÉS A PART

O. Danos.
Une journée Recherche dans le cadre d'EUROCANCER 93 se tiendra à Paris le 27 avril 1993, sous la présidence de $P$. Tambourin et M. Boiron, sur le thème "De la recherche oncologique à l'innovation thérapeutique " avec, entre autres, le patronage de I'INSERM.

\section{Programme}

- Introduction et présentation de la journée (P. Tambourin, Paris) ; • Génétique des antigènes de rejet tumoral : nouvelles perspectives pour l'immunothérapie spécifique (Th. Boon, Bruxelles); - Contrôle de l'expression génétique (M. Yaniv, Paris) ; - Oncogènes mutants : cibles pour la thérapie ( $N$. Lemoine, Londres) ; - Le couple prolifération-différenciation (L. Degos et $H$. de Thé, Paris) ; - Nouvelles cibles et nouveaux concepts en chimiothérapie anti-cancéreuse (N.W. Lobbezoo, Amsterdam) ; - Chimioprévention des cancers (A. Costa, Milan) ; - Modulation de la résistance multidrogue associée à la P-glycoprotéine des cellules tumorales (G. Atassi, Paris); - Chimio- et radioprotecteurs (M. Marty, Paris) ; - Transfert de gènes (Th. Velu, Bruxelles et T. Blankenstein, Berlin): - Anticorps monoclonaux humains (J. Banchereau, Lyon): - Conclusion (M. Boiron, Paris).

L'examen des posters et leur discussion auront lieu au moment de la pause-déjeuner.

3 posters seront sélectionnés pour présentation orale par le jury scientifique d'Eurocancer : P. Tambourin (Président), F. Calvo, H. Fridman (INSERM U.255), S. Gisselbrecht, C.-J. Larsen (INSERM U.301), A. Tavitian (INSERM U.301), A. Tavitian (INSERM U.248), Th. Tursz.

Le prix du meilleur poster sera remis en fin de journée par P. Tambourin et le comité scientifique.

Les actes de cette journée seront disponibles sur forme d'une co-édition INSERM/John Libbey Eurotext dans la collection des Colloques de I'INSERM. Pour renseignement complémentaire et inscription, s'adresser au secrétariat scientifique : M. Boiron, M. Marty, M.-C. Guédès, Centre Hayem, Hôpital SaintLouis, 1, avenue Vellefaux, 75010 Paris. Tél. : 42.06.32.60 ou 42.03.36.56 - Télécopie : 42.41.14.70.

Un symposium international sur le thème "Ochratoxicose humaine et pathologies associées en Afrique et dans les pays en voie de développement " se tiendra à Bordeaux du 3 au 7 juillet 1993 avec le soutien de I'INSERM, de I'UNEP (United Nation Environment Program) et de la Région Aquitaine. II réunira les principaux spécialistes internationaux de l'ochratoxine $A$ (mycotoxine, contaminant alimentaire) (G. Dirheimer, H. Bartsch, M. Castegnaro, T. Kuiper-Goodman, P. Galtier, P. Bach, R. Plestina, B. Hald, P. Steyn, Y. Ueno, etc.) et des équipes de chercheurs ayant mis en évidence des maladies liées à l'ochratoxicose humaine et animale dans leurs pays respectifs (Afrique, Asie). Les objectifs principaux sont les suivants:

- savoir si la néphropathie endémique des Balkans, vraisemblablement due à I'ochratoxine $A$, existe en dehors de cette aire géographique ;

- quelles sont les pathologies associées à l'ochratoxicose dans les pays en voie de développement, en comparaison avec ce qui est observé dans les Balkans :

- comment expliquer la spécificité rénale de cette mycotoxicose (néphrotoxicité et tumeurs) ;

- quels sont les moyens de prévention et/ou de traitement de l'ochratoxicose (25 à $60 \%$ au moins des personnes sont OTA-positives en Europe) ;

- comment mettre en place un réseau Nord-Sud de collaboration sur ce sujet qui concerne aussi bien les pays industrialisés que les pays sous-développés.

Les actes paraîtront pour le symposium, sous forme d'une co-édition INSERM/John Libbey Eurotext dans la collection des Colloques de I'INSERM.

Pour tout renseignement et inscription, s'adresser avant le 28 février 1993 au Pr. E. E. Creppy, Laboratoire de Toxicologie, Université de Bordeaux II, 33076 Bordeaux cedex, "Tél. : 56.91.84.07" Télécopie : 56.91.14.16. 